\title{
Correlación Espacial entre Malas hierbas en una Pradera y su Relación con la Conductividad Eléctrica Aparente del SUELO $\left(\mathrm{CE}_{\mathrm{A}}\right)^{1}$
}

\author{
Spatial Correlation Between Weeds in a Grassland and its Relationship to Soil Apparent \\ Electrical Conductivity $\left(E C_{d}\right)$
}

SIQUEIRA, G.M. ${ }^{2}$, DAFONTE DAFONTE, J. ${ }^{3}$ y VALCÁRCEL ARMESTO, M. ${ }^{3}$

\begin{abstract}
RESUMEN - El objetivo de este trabajo fue evaluar la ecología y la correlación lineal y espacial entre malas hierbas de una pradera y su relación con la conductividad eléctrica aparente del suelo $\left(\mathrm{CE}_{\mathrm{a}}, \mathrm{mS} \mathrm{m}^{-1}\right)$. La toma de muestras se hizo los dias: 23/06/2008 ( $\left.\mathrm{CE}_{\mathrm{a}}\right)$ y el 24/06/2008 (malas hierbas). Los datos fueron analizados por medio de estadística clásica y de herramientas de geoestadística. La mayoría de los datos presentaron distribución de frecuencia logarítmica, siendo necesaria la utilización del krigeado residual. Se encontraron bajos valores de correlación para las medidas de $\mathrm{CE}_{\mathrm{a}}$ con las mediciones de malas hierbas en el área de estudio, estando las mismas influidas por el relieve del área. Los mapas de variabilidad espacial mostraron que existía relación entre las malas hierbas y los valores de $\mathrm{CE}_{\mathrm{a}}\left(\mathrm{CE}_{\mathrm{a}}-\mathrm{V}\right.$ y $\left.\mathrm{CE}_{\mathrm{a}}-\mathrm{H}\right)$, principalmente con relación al número de especies de malas hierbas, ya que hay un aumento del número de especies cuando se produce disminución de los valores de $\mathrm{CE}_{\mathrm{a}}$. Los mapas de variabilidad espacial para las malas hierbas demuestran la dominancia de ciertas especies y el endemismo de otras.
\end{abstract}

Palabras-clave: geoestadística, atributos biológicos del suelo, variabilidad espacial, manejo del suelo, manejo de praderas.

\begin{abstract}
The objective of this study was to evaluate the ecology and, linear and spatial correlation between weeds in and soil apparent electrical conductivity $\left(E C_{a}, \mathrm{mS} \mathrm{m}^{-1}\right)$ in a grassland. Measurements were done in 23/06/2008 (EC $)$ and 24/06/2008 (weeds). Data were analyzed using classical statistical and geostatistical tools. Most data presented logarithmic frequency distribution, using the residual kriging being necessary. Low values of correlation measures EC measurements with weeds in the study area were found, being influenced by the topography of the area. The spatial variability maps have showed relationship between weeds and values of $E C_{a}$ $\left(E C_{a}-V\right.$ and $\left.E C_{a}-H\right)$, mainly regarding the number of weed species, as there are an increasing number of species with declining $E_{2}$ values. The maps of spatial variability for weeds demonstrate the dominance of species and endemism of other species.
\end{abstract}

Keywords: geostatistics, soil and biological attributes, spatial variability, soil management, grassland management.

\section{INTRODUCCIÓN}

En los países europeos es una práctica habitual el pastoreo en praderas cultivadas, o también, el uso de praderas para la producción de forraje de diferentes tipos. En este sentido, es necesario comprender la dinámica y la ecología de los agrosistemas de producción, para poder determinar cuáles son las mejores alternativas de manejo para el suelo con relación a la existência de malas hierbas.

1 Recebido para publicação em 19.11.2014 e aprovado em 16.6.2015.

2 Universidade Federal do Maranhão, São Luís-MA, Brasil, <gleciosiqueira@hotmail.com>; ${ }^{3}$ Escuela Politécnica Superior, Universidade de Santiago de Compostela, Lugo, Espanha. 
De acuerdo con Schaffrath et al. (2007) el manejo del suelo interfiere sobre la variabilidad espacial de malas hierbas en suelos cultivados con praderas. Mosquera Losada et al. (1999) describen que algunas especies de malas hierbas se mantienen inertes en el sistema agrícola hasta que tienen condiciones favorables para su pleno desarrollo.

Por otra parte, San Miguel Ayanz (2007), describe que el poder de desarrollo de una especie de mala hierba está condicionado por su capacidad de competición, que en muchos casos es variable en función de las características extrinsecas que le son ofrecidas durante su desarrollo.

Distintos autores han encontrado diferentes patrones espaciales de desarrollo vegetativo para las malas hierbas, no habiendo una constante que permita decir que característica del manejo de suelo es la que más incluye sobre su manifestación en el campo (Colbach et al., 2000; Jurado-Expósito et al., 2004, 2009).

Shiratsuchi et al. (2004) describen que el comportamiento de las especies de malas hierbas en campo no ocurre de manera uniforme, en este sentido Lass \& Callihan (1993) y Colliver et al. (1996) proponen que el muestreo de las malas hierbas en campo sea realizado junto con un mapeado del contorno de las zonas de mayor ocurrencia de las especies de malas hierbas, generando mapas de polígonos de infestación.

Según Lesch et al. (2000), Lesch (2005), Rhoades et al. (1976) y McNeill (1980) la conductividad eléctrica aparente del suelo ha sido bastante utilizada en los últimos años ya que tiene una fuerte relación con diferentes propiedades del suelo, como: porosidad, concentración de electrólitos disueltos, textura, cantidad y composición de los coloides (arcilla y materia orgánica), contenido de materia orgánica y contenido de agua en el solo.

De esta forma, el objetivo de este trabajo fue evaluar la ecología y correlación lineal y espacial entre malas hierbas de una pradera y la conductividad eléctrica aparente del suelo $\left(\mathrm{CE}_{\mathrm{a}}, \mathrm{mS} \mathrm{m}^{-1}\right)$, para una parcela situada en Castro de Ribeiras de Lea (Lugo, España).

\section{MATERIAL Y METODOS}

El área de estudio ocupa 6 ha y está localizada en Castro de Ribeiras de Lea (Lugo, España). Las coordenadas geográficas son: 4309'49" N y 7०29'47" W, con una altitud media de $410 \mathrm{~m}$ y pendiente del 2\% (Figura 1 ).

El clima de la región, según la clasificación climática de Köppen, es del tipo templado (Cfb), con verano cálido y sin estación seca, con una precipitación media anual de $930 \mathrm{~mm}$. El suelo de la parcela es un Cambisol gleyco (FAOISRIC, 1994) con problemas de hidromorfia estacionales. En los últimos años el área de estudio fue cultivada con pradera permanente para producción de ensilado.

La conductividad eléctrica aparente del suelo $\left(\mathrm{CE}_{\mathrm{a}}\right)$ fue medida el 23/06/2008 utilizando un equipo de inducción electromagnética EM38-DD (Geonics, 2005) que consta de dos dipolos colocados perpendicularmente, cuya curva de respuesta relativa demuestra una mayor sensibilidad del aparato hasta $0,4 \mathrm{~m}$ en el dipolo horizontal y de $1,5 \mathrm{~m}$ en el dipolo vertical (Geonics, 2005). La conductividad eléctrica en el dipolo horizontal $\left(\mathrm{CE}_{\mathrm{a}}{ }^{-}\right.$ $\mathrm{H}, \mathrm{mS} \mathrm{m}^{-1}$ ) y la conductividad eléctrica en el dipolo vertical $\left(\mathrm{CE}_{\mathrm{a}}-\mathrm{V}, \mathrm{mS} \mathrm{m}^{-1}\right)$ y fue medida en 1859 puntos de muestreo (Figura 2), usando un GPS RTK para realizar el georeferenciamiento de las medidas. El EM38-DD se instaló en un carro construido con materiales plásticos para evitar interferencias con las medidas, diseñado por nuestro grupo de investigación.

E1 análisis de las especies de malas hierbas presentes en el área de estudio fue realizado el 24/06/2008 utilizando un circulo con $0,5 \mathrm{~m}$ de diámetro $\left(0,196 \mathrm{~m}^{2}\right)$, lanzado al azar al lado de cada uno de los puntos de muestreo, considerando la técnica de detección manual (Lutman \& Perry, 1999). El muestreo del número de malas hierbas fue realizado en los 40 puntos de muestreo optimizados (Figura 2) en el día 23/06/2008, con el software ESAP-RSSD 2.35 que utiliza los valores $\mathrm{CE}_{\mathrm{a}}$ para diseñar un esquema de muestreo, basándose en un algoritmo de superficie de respuesta múltiple (Lesch, 2000).

La identificación de las especies de malas hierbas presentes en el área de estudio se 

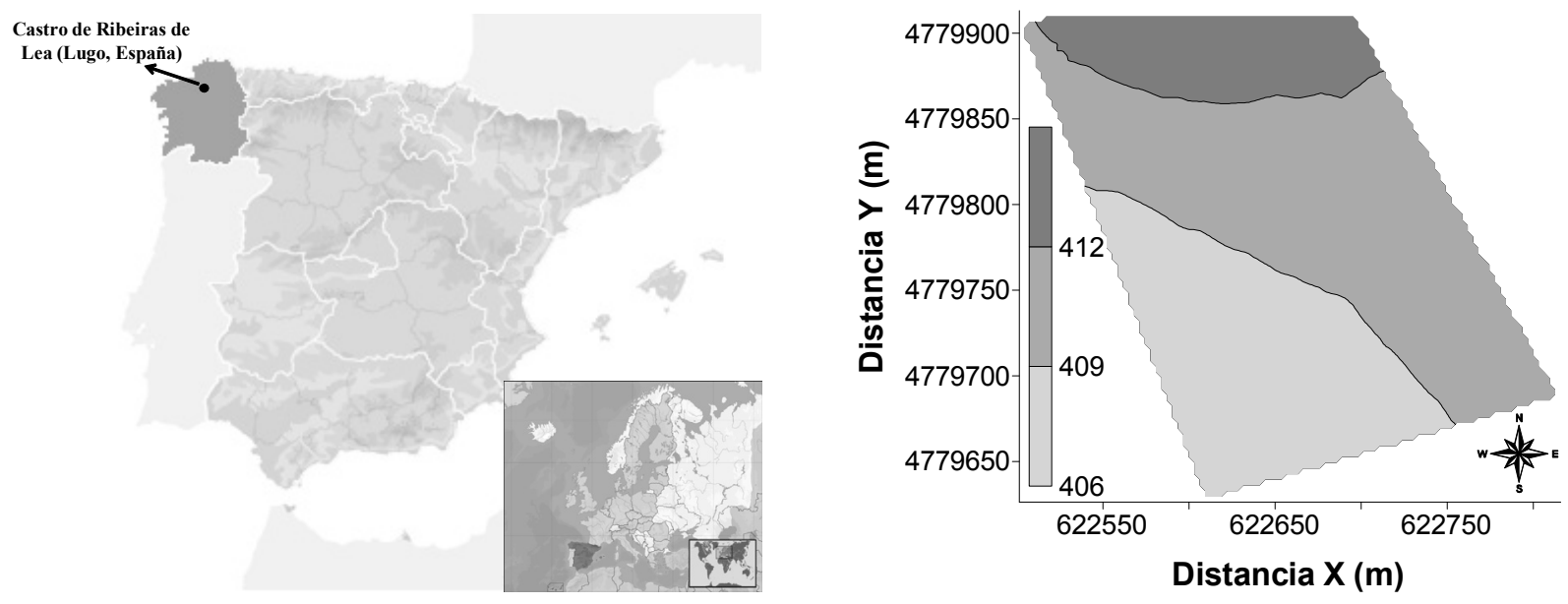

Figura 1 - Localización geográfica del área de estudio (A) y mapa de elevación digital (B).

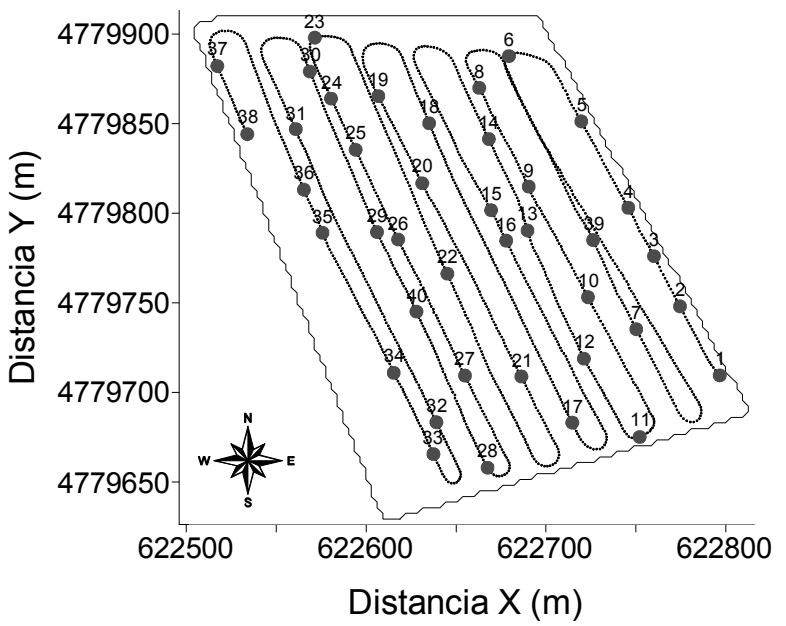

Figura 2 - Esquema de medidas de la conductividad eléctrica aparente del suelo medida por inducción electromagnética (línea continua) y el esquema de muestreo de los 40 puntos de toma de muestra de malas hierbas (círculos) el $23 / 06 / 2008$.

realizó usando el Atlas de Malas Hierbas (Villarias, 2000). Durante el proceso de análisis de los datos, el trébol violeta (Trifolium pratense) y el trébol blanco (Trifolium repens) fueron agrupados (Trifolium sp.). También se encontraron otras especies de malas hierbas en el área de estudio, pero que se presentaban con menor densidad y con un comportamiento endémico; por esta razón en el presente estudio, se prefirió contabilizar estas especies en una única categoría llamada otras especies: raigrás italiano (Lolium multiflorum), hierba cana (Senecio vulgaris) y mostaza negra (Brassica nigra).
Los análisis estadísticos fueron realizados para determinación de los principales momentos estadísticos: media, varianza, desviación estándar, coeficiente de variación, asimetría, curtosis y la desviación máxima en relación a la distribución de frecuencia normal por medio del test de KolmogorovSmirnov con probabilidad del error de $\mathrm{p}<0,01$.

Con el objetivo de determinar la correlación lineal entre la conductividad eléctrica aparente del suelo $\left(\mathrm{CE}_{\mathrm{a}}\right)$ medida en los dipolos vertical $\left(\mathrm{CE}_{\mathrm{a}}-\mathrm{V}\right)$ y el dipolo horizontal $\left(\mathrm{CE}_{\mathrm{a}}-\mathrm{H}\right)$ y las propiedades del suelo y planta estudiadas, fueron construidas matrices de correlación entre los pares de datos. La clasificación de Santos (2007) fue utilizada para determinar el valor de la correlación entre los pares de datos: $|r|=1$ : perfecta; $0,8 \leq|r|<1$ : fuerte; $0,5 \leq|r|<0,8$ : moderada; $0,1 \leq|r|<0,5$ : débil; $0<|r|<0,1$ : débilmente y $r=0$ : nula.

En este trabajo se ha efectuado el análisis geoestadístico construyendo y modelando semivariogramas experimentales y determinando los valores para los puntos no muestreados por medio del krigeado ordinario. El análisis inicial demostró que algunas variables presentaban tendencia, en cuyo caso se podrian utilizar el krigeado ordinario residual o el krigeado universal. En este caso se utilizó el krigeado ordinario residual, previamente se realizó la retirada de tendencia utilizando los siguientes modelos: 


\section{Lineal}

$$
m(x)=A_{0}+A_{1} x+A_{2} y+A_{3} x y
$$

\section{Cuadrática o parabólica}

$$
m(x)=A_{0}+A_{1} x+A_{2} y+A_{3} x^{2}+A_{4} y^{2}+A_{5} x y
$$

Cúbica

$$
m(x)=A_{0}+A_{1} x+A_{2} y+A_{3} x^{2}+A_{4} y^{2}+A_{5} x y+A_{6} x^{3}+A_{7} y^{3}+A_{8} x^{2} y+A_{9} x y^{2} \quad \text { (ec. 3) }
$$

De esta manera, con la retirada de la tendencia fue posible determinar el semivariograma experimental de los residuos, ajustando un modelo de semivariograma mediante validación cruzada utilizando el software Progeoestat (Vieira et al., 2002).

Para la construcción de los mapas de isolineas se empleó el software Surfer 7.0 (Golden Software, 1999) considerando los valores determinados por medio del krigeado residual donde se supone conocida la deriva $m(x)$. La deriva espacial se estimó mediante el método de mínimos cuadrados, los residuos se calcularon mediante la Ecuación 4:

$$
R(x)=Z(x)+m(x)
$$

donde $R(x)$ - son los residuos en la localización $\mathrm{x} ; Z(x)$ - es el valor de la variable en la localización $\mathrm{x}$.

El grado de dependencia espacial (GD, \%) fue determinado de acuerdo con Cambardella et al. (1994): $\mathrm{GD}=\mathrm{C}_{0} / \mathrm{C}_{0}+\mathrm{C}_{1}{ }^{*} 100$.

\section{RESULTADOS Y DISCUSIÓN}

Los parámetros estadísticos de la densidad de malas hierbas: Dactylis glomerata (dáctilo), Trifolium sp. (trébol), Bellis perennis (margarita), Plantago lanceolata (llantén menor), Taraxacum officinale Weber (diente de león), Achillea millefolium (milenrama), otras especies, densidad de individuos y número de especies en el área de estudio son presentados en la Tabla 1.

Los valores de coeficiente de variación $(\mathrm{CV}, \%)$ muestran que la mayoría de las malas hierbas presentan valores medios de $\mathrm{CV}$ (12-60\%) (Trifolium sp., Bellis perennis, Plantago lanceolata, Taraxacum officinale,
Achillea millefolium, densidad de individuos y el número de especies), con excepción del Dactylis glomerata $(\mathrm{CV}=64,01 \%)$ y otras especies $(\mathrm{CV}=67,47 \%)$ que presentan valores de CV elevados ( $\geq 60 \%$ ), según la clasificación de Warrick \& Nielsen (1980). Schaffrath et al. (2007) estudiando la variabilidad espacial de malas hierbas en diferentes sistemas de manejo del suelo encontraron valores de CV entre $86,05 \%$ y $168,85 \%$ para la siembra directa y entre $74,73 \%$ y $81,72 \%$ para el cultivo convencional.

La desviación máxima en relación a la distribución de frecuencia normal por medio de test estadístico de Kolgomorov-Smirnov (D) muestra que el Dactylis glomerata $(\mathrm{D}=0,160 \mathrm{n})$, Achillea millefolium $(\mathrm{D}=0,311 \mathrm{n})$ y la densidad de individuos $(D=0,113 \mathrm{n})$ presentan una distribución de frecuencia normal (Tabla 1). Los demás datos presentan una distribución de frecuencia lognormal (Trifolium sp., Bellis perennis, Plantago lanceolata, Taraxacum officinale, otras especies y el número de especies).

Isaaks \& Srivastava (1989) describen que el coeficiente de asimetría es más sensible a los valores extremos, cuando se compara con otros parámetros estadísticos como la media, mediana y desviación estándar, en este caso un único valor extremo puede influir de forma muy significativa en el valor del coeficiente de asimetría, ya que la desviación entre cada valor y la media es elevada a la tercera potencia.

Por medio del valor medio (Tabla 1) se comprueba que el Dactylis glomerata es la especie de mala hierba que aparece con mayor frecuencia en el área de estudio, con una media de 24 plantas por $\mathrm{m}^{2}$, el menor valor fue 
encontrado para Achillea millefolium que presenta una media de 9 plantas por $\mathrm{m}^{2}$. El número de especies de malas hierbas presentes en el área de estudio varía de 1 especie por $\mathrm{m}^{2}$ hasta alcanzar en algunas zonas del área de estudio un valor de 6 especies por $\mathrm{m}^{2}$, teniendo un valor medio de 4 especies por $\mathrm{m}^{2}$ en el área. La densidad de individuos demuestra que en el área de estudio se han encontrado una media de 52 plantas por $\mathrm{m}^{2}$, presentando un valor mínimo y máximo de 26 plantas por $\mathrm{m}^{2}$ y 77 plantas por $\mathrm{m}^{2}$, respectivamente.

Se obtienen valores de media próximos para la $\mathrm{CE}_{\mathrm{a}}-\mathrm{V}$ y $\mathrm{CE}_{\mathrm{a}}-\mathrm{H}$ (Tabla 2), debido a la interferencia del nivel freático sobre la lecturas con el equipo EM38-DD, ya que elevados valores de humedad en suelo permiten menores diferencias entre los valores medidos por los dipolos (Lesch, 2000).

La matriz de correlación lineal (Tabla 2) muestra que la mayoría de las correlaciones entre la conductividad eléctrica aparente $\left(\mathrm{CE}_{\mathrm{a}}{ }^{-}\right.$ $\mathrm{V}$ y $\left.\mathrm{CE}_{\mathrm{a}}-\mathrm{H}\right)$ y las especies de malas hierbas (Dactylis glomerata, Trifolium sp., Bellis perennis, Plantago lanceolata, Taraxacum officinale, Achillea millefolium, otras especies, densidad de individuos y número de especies) son clasificadas como débiles $(r=0,1-0,5)$ o muy débiles $(r=0,0-0,1)$, según la clasificación de Santos (2007).

La correlación lineal más alta entre la conductividad eléctrica aparente del suelo

Tabla 1 - Parámetros estadísticos para las principales especies malas hierbas presentes en el área de estudio

\begin{tabular}{|c|c|c|c|c|c|c|c|c|c|c|}
\hline Variable & Unidad & $\mathrm{N}$ & Mín & Máx & Media & Varianza & $\mathrm{CV}$ & Asim. & Curt. & $\mathrm{D}$ \\
\hline Dactylis glomerata & \multirow{8}{*}{ plantas $\mathrm{m}^{-2}$} & 30 & 5 & 51 & 24 & 229,00 & 64,01 & 0,503 & $-0,986$ & $0,160 \mathrm{n}$ \\
\hline Trifolium $\mathrm{sp}$. & & 18 & 7 & 26 & 10 & 26,50 & 50,47 & 0,686 & $-0,124$ & $0,246 \mathrm{Ln}$ \\
\hline Bellis perennis & & 25 & 5 & 31 & 13 & 32,40 & 43,15 & 0,286 & $-0,107$ & $0,191 \mathrm{Ln}$ \\
\hline Plantago lanceolata & & 24 & 4 & 31 & 13 & 43,20 & 52,41 & 1,029 & 1,087 & $0,209 \mathrm{Ln}$ \\
\hline Taraxacum officinale & & 22 & 5 & 20 & 10 & 20,10 & 43,29 & 0,749 & 0,209 & $0,290 \mathrm{Ln}$ \\
\hline Achillea millefolium & & 17 & 1 & 15 & 9 & 18,80 & 48,61 & 0,534 & $-1,416$ & $0,311 \mathrm{n}$ \\
\hline Otras especies & & 27 & 2 & 36 & 13 & 73,00 & 67,47 & 1,737 & 4,393 & $0,218 \mathrm{Ln}$ \\
\hline Densidad de individuos & & 40 & 26 & 77 & 52 & 185,00 & 26,00 & 0,308 & $-0 ., 761$ & $0,113 n$ \\
\hline Número de especies & especies $\mathrm{m}^{-2}$ & 40 & 1 & 6 & 4 & 1,60 & 35,30 & $-0,403$ & $-0,302$ & $0,204 \mathrm{Ln}$ \\
\hline $\mathrm{CE}_{\mathrm{a}}-\mathrm{V}$ & \multirow{2}{*}{$\mathrm{mS} \mathrm{m}^{-3}$} & 1859 & 4,13 & 20,13 & 11,21 & 6,12 & 22,07 & 0,485 & $-0,243$ & $0,071 \mathrm{Ln}$ \\
\hline $\mathrm{CE}_{\mathrm{a}}-\mathrm{H}$ & & 1859 & 6,63 & 20,00 & 12,12 & 3,22 & 14,81 & 0,839 & 1,285 & $0,092 \mathrm{Ln}$ \\
\hline
\end{tabular}

N: número de puntos; Mín.: valor mínimo; Máx.: valor máximo; CV: coeficiente de variación; Asim.: coeficiente de asimetría; Curt.: coeficiente de curtosis; D: desviación máxima en relación a la distribución de frecuencia normal por medio del test de Kolmogorov-Smirnov con probabilidad del error de $\mathrm{p}<0,01$; n: normal; Ln: Lognormal.

Tabla 2 - Matriz de correlación lineal entre la conductividad eléctrica aparente del suelo ( $\left.\mathrm{CE}_{\mathrm{a}}\right)$ y las malas hierbas

\begin{tabular}{|c|c|c|c|c|c|c|c|c|c|c|c|}
\hline & $\mathrm{CE}_{\mathrm{a}}-\mathrm{V}$ & $\mathrm{CE}_{\mathrm{a}}-\mathrm{H}$ & $\begin{array}{c}\text { Dactylis } \\
\text { glomerata }\end{array}$ & Trifolium $\mathrm{sp}$. & $\left|\begin{array}{c}\text { Bellis } \\
\text { perennis }\end{array}\right|$ & $\begin{array}{l}\text { Plantago } \\
\text { lanceolata }\end{array}$ & $\begin{array}{c}\text { Taraxacum } \\
\text { officinale }\end{array}$ & $\begin{array}{c}\text { Achillea } \\
\text { millefolium }\end{array}$ & $\begin{array}{c}\text { Otras } \\
\text { especies }\end{array}$ & \begin{tabular}{|c} 
Densidad \\
de \\
individuos
\end{tabular} & $\begin{array}{l}\text { Número } \\
\text { de } \\
\text { especies }\end{array}$ \\
\hline $\mathrm{CE}_{\mathrm{a}}-\mathrm{V}$ & 1,000 & & & & & & & & & & \\
\hline $\mathrm{CE}_{\mathrm{a}}-\mathrm{H}$ & 0,748 & 1,000 & & & & & & & & & \\
\hline Dactylis glomerata & 0,273 & $-0,219$ & 1,000 & & & & & & & & \\
\hline Trifolium $\mathrm{sp}$. & 0,147 & 0,016 & $-0,078$ & 1,000 & & & & & & & \\
\hline Bellis perennis & $-0,352$ & $-0,370$ & $-0,374$ & $-0,208$ & 1,000 & & & & & & \\
\hline Plantago lanceolata & $-0,080$ & 0,160 & $-0,508$ & $-0,078$ & 0,045 & 1,000 & & & & & \\
\hline Taraxacum officinale & \begin{tabular}{|l|}
$-0,157$ \\
\end{tabular} & 0,032 & $-0,458$ & $-0,090$ & 0,102 & 0,070 & 1,000 & & & & \\
\hline Achillea millefolium & $-0,193$ & $-0,005$ & $-0,231$ & $-0,056$ & 0,094 & $-0,173$ & $-0,037$ & 1,000 & & & \\
\hline Otras especies & $-0,034$ & 0,488 & $-0,554$ & $-0,147$ & $-0,145$ & 0,027 & 0,140 & 0,083 & 1,000 & & \\
\hline Densidad de individuos & $-0,112$ & $-0,161$ & 0,890 & $-0,246$ & $-0,605$ & $-0,161$ & 0,255 & 0,336 & 0,450 & 1,000 & \\
\hline Número de especies & $-0,278$ & $-0,155$ & $-0,627$ & 0,342 & 0,489 & 0,252 & 0,219 & 0,227 & 0,042 & $-0,780$ & 1,000 \\
\hline
\end{tabular}


$\left(\mathrm{CE}_{\mathrm{a}}-\mathrm{V}\right.$ y $\left.\mathrm{CE}_{\mathrm{a}}-\mathrm{H}\right)$ y las especies de malas hierbas que se ha encontrado fue: entre $\mathrm{CE}_{\mathrm{a}}-\mathrm{H}$ x Otras especies $(r=0,488)$ indicando que cuanto mayor es el número de otras especies de malas hierbas en el área mayores son los valores de $\mathrm{CE}_{\mathrm{a}}-\mathrm{H}$; y entre $\mathrm{CE}_{\mathrm{a}}-\mathrm{V} \mathrm{x}$ Bellis perennis $(\mathrm{r}=-0,352)$ mostrando que cuanto mayores son los valores de $\mathrm{CE}_{\mathrm{a}}-\mathrm{V}$ menor es la cantidad de Bellis perennis presente en el área de estudio. Los valores de correlación lineal entre la $\mathrm{CE}_{\mathrm{a}}$ $\mathrm{V}$ y las especies de malas hierbas son negativos para la mayoría de las correlaciones posibles, con excepción de la relación entre $\mathrm{CE}_{\mathrm{a}}-\mathrm{V}$ x Dactylis glomerata $(\mathrm{r}=0,273)$ y $\mathrm{CE}_{\mathrm{a}}-$ $\mathrm{V} x$ Trifolium sp. $(\mathrm{r}=0,147)$.

La correlación lineal entre el Dactylis glomerata y el Trifolium sp. con las demás especies de malas hierbas (Bellis perennis, Plantago lanceolata, Taraxacum officinale, Achillea millefolium y otras especies) es negativa, indicando que cuanto mayor es la ocurrencia de plantas de Dactylis glomerata y el Trifolium sp. en el área, menor es la ocurrencia de las demás especies estudiadas (Bellis perennis, Plantago lanceolata, Taraxacum officinale, Achillea millefolium y otras especies). Según San Miguel Ayanz (2008) el Dactylis glomerata es una planta muy competitiva, al contrario el Trifolium sp. que es una especie poco competitiva, por el contrario Mosquera Losada (1999) describe que en algunas situaciones el Trifolium sp. se mantiene inerte hasta que encuentra condiciones aptas para su desarrollo, lo que le permite una mayor competitividad frente a otras especies de malas hierbas.
La densidad de individuos presenta correlación negativa con $\mathrm{CE}_{\mathrm{a}}-\mathrm{V}(\mathrm{r}=-0,112)$, $\mathrm{CE}_{\mathrm{a}}-\mathrm{H}(\mathrm{r}=-0,161)$, Trifolium sp. $(\mathrm{r}=-0,246)$, Bellis perennis $(\mathrm{r}=-0,605)$, Plantago lanceolata $(\mathrm{r}=-0,161)$ y el número de especies $(\mathrm{r}=-0,780)$, eso se debe principalmente a que el Dactylis glomerata es la especie de mala hierba más abundante en el área, demostrando su elevada competitividad cuando se compara con las demás especies de malas hierbas presentes en el área.

La Tabla 3 presenta los parámetros de ajuste del semivariograma para las especies de malas hierbas (Dactylis glomerata, Trifolium sp., Bellis perennis, Plantago lanceolata, Taraxacum officinale, Achillea millefolium, otras especies, densidad de individuos y número de especies) y para la conductividad eléctrica aparente del suelo $\left(\mathrm{CE}_{\mathrm{a}}-\mathrm{V}\right.$ y $\left.\mathrm{CE}_{\mathrm{a}}-\mathrm{H}\right)$.

El grado de dependencia espacial (GD, \%) para la densidad de individuos $(\mathrm{GD}=11,11 \%)$, el Dactylis glomerata (GD $=13,67 \%$ ) y Plantago lanceolata $(\mathrm{GD}=19,05 \%)$ según la clasificación de Cambardella et al. (1994) es elevado (GD $\leq 25 \%$ ), las demás especies de malas hierbas estudiadas presentaron un grado moderado de dependencia espacial entre las muestras (GD $=25-75 \%$ ).

E1 modelo esférico se ajustó a los semivariogramas del Dactylis glomerata, Bellis perennis, Achillea millefolium y al número de especies. El modelo exponencial se ajustó a las siguientes especies de malas hierbas:

Tabla 3 - Parámetros de ajuste del semivariograma las especies de malas hierbas más frecuentes en el área de estudio

\begin{tabular}{|c|c|c|c|c|c|}
\hline Variable & Modelo & $\mathrm{C}_{0}$ & $\mathrm{C}_{1}$ & $\mathrm{a}(\mathrm{m})$ & GD $(\%)$ \\
\hline Dactylis glomerata & Esférico & 1,90 & 12,00 & 210 & 13,67 \\
\hline Trifolium sp. - Residuos & Exponencial & 0,85 & 0,65 & 30 & 56,67 \\
\hline Bellis perennis - Residuos & Esférico & 0,85 & 1,65 & 50 & 34,00 \\
\hline Plantago lanceolata - Residuos & Exponencial & 0,40 & 1,70 & 40 & 19,05 \\
\hline Taraxacum officinale - Residuos & Exponencial & 0,30 & 0,75 & 40 & 28,57 \\
\hline Achillea millefolium & Esférico & 0,30 & 0,40 & 80 & 42,86 \\
\hline Otras especies - Residuos & Exponencial & 1,00 & 1,60 & 50 & 38,46 \\
\hline Densidad de individuos & Exponencial & 20,00 & 160,00 & 60 & 11,11 \\
\hline Número de especies - Residuos & Esférico & 0,83 & 0,83 & 85 & 50,00 \\
\hline Log $\mathrm{CE}_{\mathrm{a}}-\mathrm{V}$ Residuos & Esférico & 0,001 & 0,01 & 130 & 9,09 \\
\hline Log $\mathrm{CE}_{\mathrm{a}}-\mathrm{H}$ Residuos & Esférico & 0,001 & 0,05 & 130 & 1,96 \\
\hline
\end{tabular}

$\mathrm{C}_{0}$ : efecto pepita; $\mathrm{C}_{1}$ : varianza estructural; a: alcance (m); GD: grado de dependencia espacial (\%). 
Trifolium sp., Plantago lanceolata, Taraxacum officinale, otras especies y densidad de individuos.

Schaffrath et al. (2007) estudiando la variabilidad espacial de malas hierbas en diferentes sistemas de manejo del suelo ajustaron el modelo esférico y exponencial indistintamente al tipo de manejo. JuradoExpósito et al. (2009) estudiando también la variabilidad espacial de malas hierbas de dos áreas de la Provincia de Córdoba (España) cultivadas con trigo, ajustaron el modelo esférico al $80 \%$ de las especies de malas hierbas, y el modelo exponencial al resto de las especies de malas hierbas.

Jurado-Expósito et al. (2004) estudiando la variabilidad espacial y temporal de correhuela (Convolvulus arvensis) en un área de 1,6 ha en Sevilla (España) cultivada con trigo en los años 1999 y 2001 y para cultivo de girasol en los años de 2000 y 2002 ajustaron el modelo esférico en todo el periodo de estudio. Colbach et al. (2000) estudiando la variabilidad espacial de malas hierbas en un área cultivada con girasol de 1993-1997 en Morris (Minnesota, EE.UU.) también ajustaron el modelo esférico para todas las especies de malas hierbas en todo el periodo de estudio.

También ajustamos el modelo esférico a los datos de los residuos de $\mathrm{CE}_{\mathrm{a}}-\mathrm{V}$ y $\mathrm{CE}_{\mathrm{a}}-\mathrm{H}$, con un alcance de $13 \mathrm{~m}$ y con un elevado grado de dependencia espacial entre la muestras, según la clasificación de Cambardella et al. (1994).

El mayor valor de alcance (a, en $\mathrm{m}$ ) se ajustó para el Dactylis glomerata con $210 \mathrm{~m}$ y el menor valor de alcance se ajustó para Trifolium sp. con $30 \mathrm{~m}$. En este caso podemos decir que hay una relación entre el alcance la capacidad de dispersión de cada especie y también con la existencia de tendencia o no en los semivariogramas residuales presentados en la Tabla 3 .

Las malas hierbas presentaron en general unos valores bajos para el alcance $(\mathrm{a}, \mathrm{m})$ variando entre $30 \mathrm{~m}$ y $85 \mathrm{~m}$, con excepción del Dactylis glomerata $(210 \mathrm{~m})$. Schaffrath et al. (2007) estudiando la variabilidad espacial de malas hierbas en diferentes sistemas de manejo del suelo (siembra directa y cultivo convencional) en Campo Mourão (Paraná,
Brasil) encontraron valores de alcance entre $9 \mathrm{~m}$ y $20 \mathrm{~m}$.

En el estudio de Schaffrath et al. (2007) las parcelas experimentales tenian un tamaño reducido $(21 \mathrm{~m} \times 65 \mathrm{~m})$ lo que provoca que los valores de alcance sean bajos, cuando se comparan con los valores de alcance de las malas hierbas encontrados en este estudio. Colbach et al. (2000) ajustaron valores de alcance (a) variando entre $9 \mathrm{~m}$ y $47 \mathrm{~m}$ para malas hierbas en un experimento con girasol desde 1993-1997. Jurado-Expósito et al. (2009) ajustaron los valores de alcance (a) entre 22,7 m y 49,7 m estudiando la variabilidad espacial de malas hierbas en Córdoba (España) para un cultivo de trigo.

Se han encontrado valores bajos de efecto pepita $\left(\mathrm{C}_{0}\right.$, Tabla 3$)$ para todas las propiedades estudiadas (Dactylis glomerata, Trifolium sp., Bellis perennis, Plantago lanceolata, Taraxacum officinale, Achillea millefolium, otras especies, densidad de individuos y número de especies). De acuerdo con Siqueira et al. (2008) el efecto pepita $\left(\mathrm{C}_{0}\right)$ indica la discontinuidad espacial entres las muestras, así cuanto mayores sean los valores de efecto pepita $\left(\mathrm{C}_{0}\right)$ mayor será la variabilidad no detectada durante el proceso de muestreo del suelo. La densidad de individuos presentó el mayor valor de efecto pepita $\left(C_{0}=20\right)$, indicando que si se hubiera muestreado un mayor número de puntos a menores distancias, se hubiera conseguido detectar con mayor precisión la variabilidad espacial a cortas distancias de la densidad de individuos en el área.

Los mapas de variabilidad espacial para las malas hierbas en estudio que se obtuvieron por krigeado ordinario y krigeado residual se presentan en la Figura 3. E1 mapa de variabilidad espacial del Dactylis glomerata (Figura 3) presenta semejanzas con los mapas de variabilidad espacial de la conductividad eléctrica aparente $\left(\mathrm{CE}_{\mathrm{a}}-\mathrm{V}\right.$ y CE $\mathrm{CE}_{\mathrm{a}}$ $\mathrm{H}$, Figura 4), mostrando que el mayor número de plantas por $\mathrm{m}^{2}$ del Dactylis glomerata están presentes en las áreas con mayor contenido de humedad, aunque su coeficiente de correlación sea bajo. San Miguel Ayanz (2008) describe que Dactylis glomerata es muy tolerante a la humedad y poco tolerante a la sequía. 


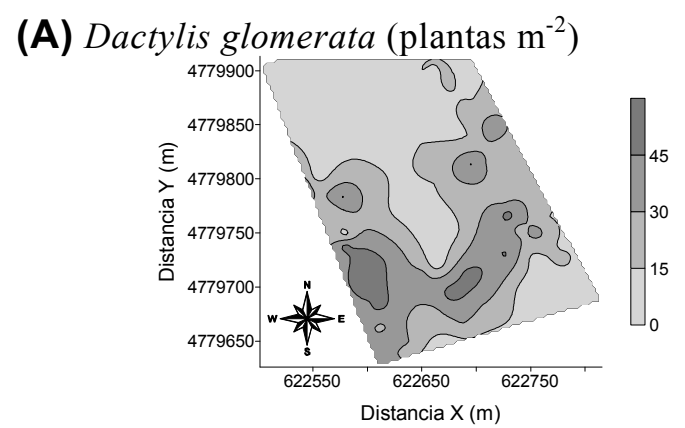

(C) Bellis perennis (plantas $\mathrm{m}^{-2}$ )

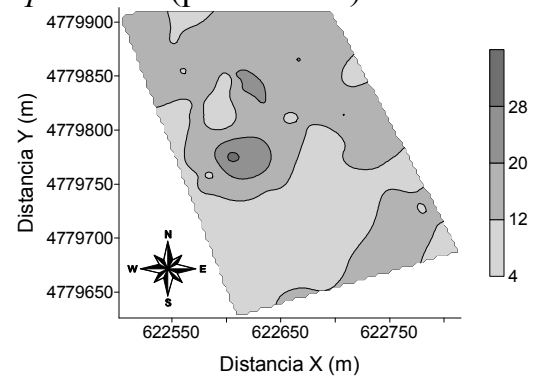

(E) Taraxacum officinale (plantas $\mathrm{m}^{-2}$ )

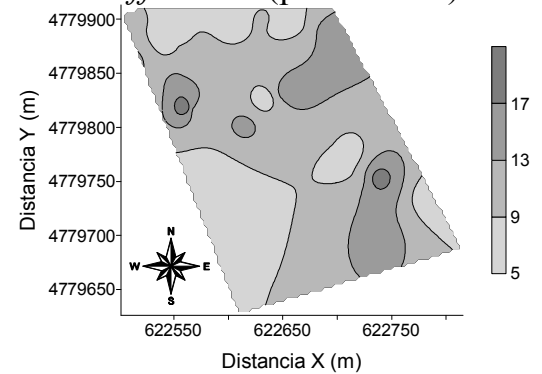

(G) Otras especies (plantas $\mathrm{m}^{-2}$ )

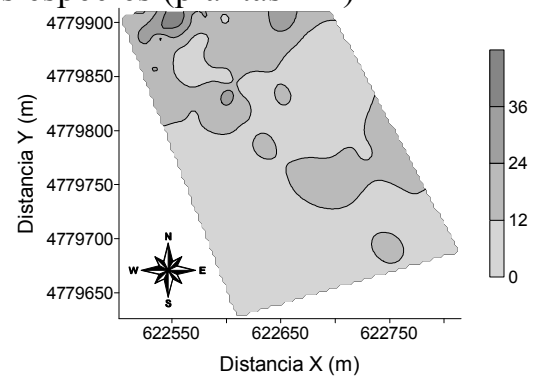

(B) Trifolium sp. (plantas $\left.\mathrm{m}^{-2}\right)$

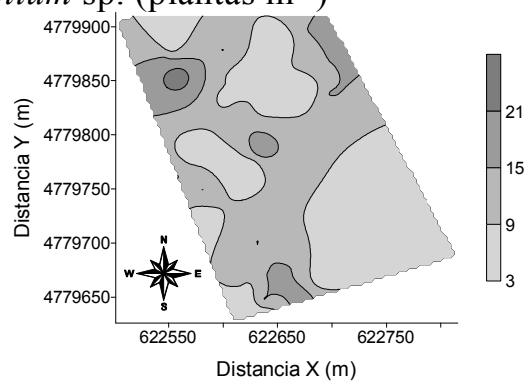

(D) Plantago lanceolata (plantas $\mathrm{m}^{-2}$ )

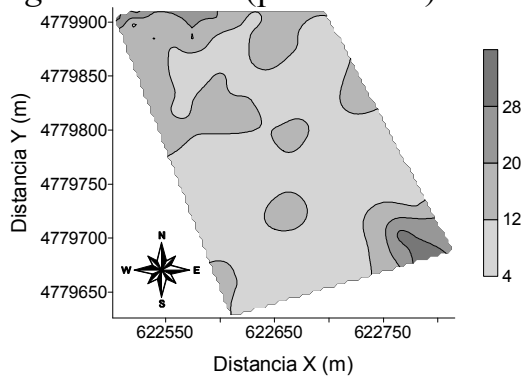

(F) Achillea millefolium (plantas $\mathrm{m}^{-2}$ )

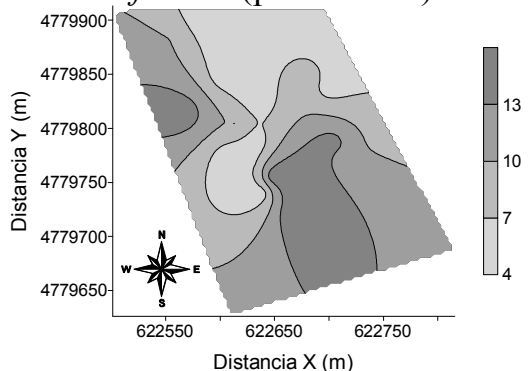

(H) Densidad de individuos (plantas $\mathrm{m}^{-2}$ )

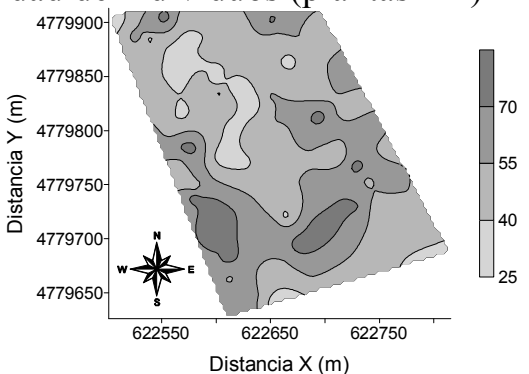

(I) Número de especies (especies $\mathrm{m}^{-2}$ )

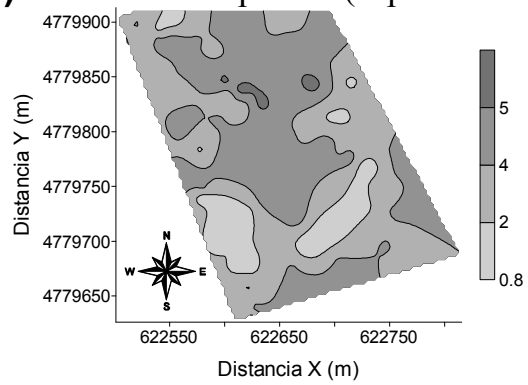

Figura 3 - Mapas de variabilidad espacial de las especies de malas hierbas: Dactylis glomerata (A); Trifolium sp. (B); Bellis perennis (C); Plantago lanceolata (D); Taraxacum officinale (E); Achillea millefolium (F); Otras especies (G); Densidad de individuos (H) y número de especies (I). 

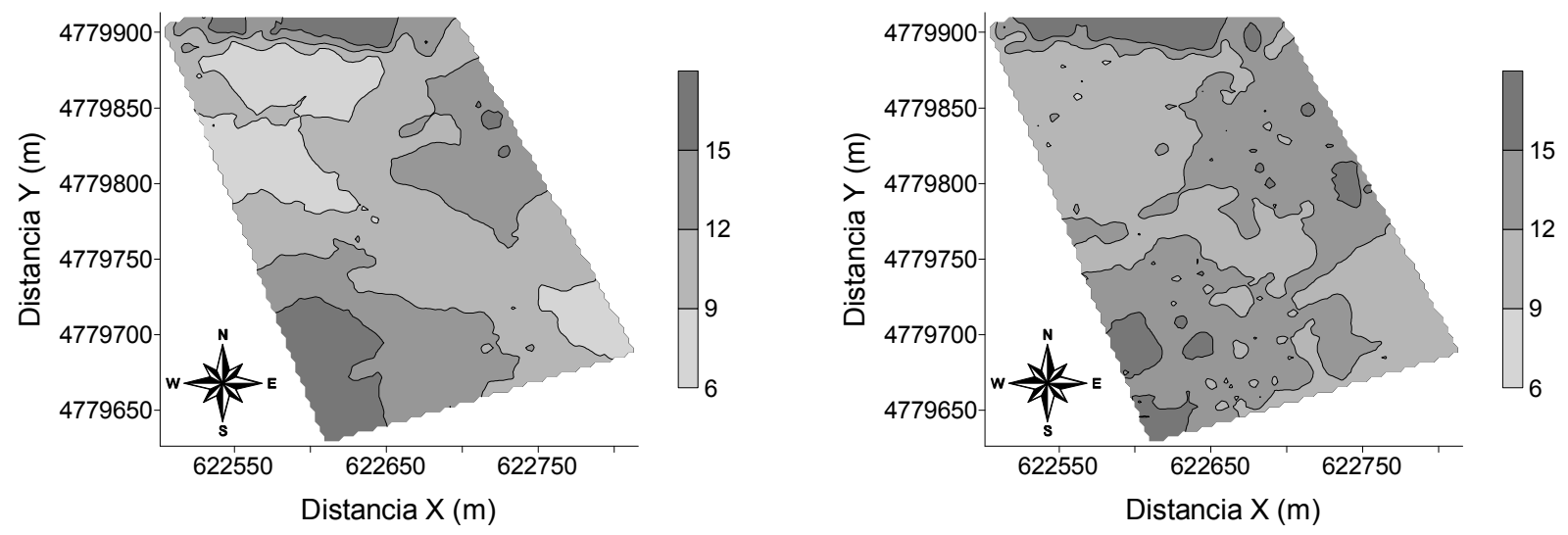

Figura 4 - Mapas de variabilidad espacial para de $\mathrm{CE}_{\mathrm{a}}-\mathrm{V}$ (A) y $\mathrm{CE}_{\mathrm{a}}-\mathrm{H}$ (B) en el día 23/06/2008.

El mapa de variabilidad espacial del Trifolium sp. (plantas por $\mathrm{m}^{2}$ ) no presenta ninguna relación con los demás mapas de variabilidad espacial $\left(\mathrm{CE}_{\mathrm{a}}-\mathrm{V}, \mathrm{CE}_{\mathrm{a}}-\mathrm{H}\right.$, Dactylis glomerata, Bellis perennis, Plantago lanceolata, Taraxacum officinale, Achillea millefolium, otras especies, densidad de individuos y número de especies) El mapa de variabilidad espacial de Bellis perennis, Plantago lanceolata y otras especies (plantas por $\mathrm{m}^{2}$ ) muestra que la parte sur del área presenta el menor número de plantas por $\mathrm{m}^{2}$, coincidiendo con el mapa de variabilidad espacial de la conductividad eléctrica aparente $\left(\mathrm{CE}_{\mathrm{a}}-\mathrm{V}\right.$ y $\mathrm{CE}_{\mathrm{a}}-\mathrm{H}$, Figura 4), y con el mapa topográfico del área (Figura 1B).

El Taraxacum officinale presenta un mapa de variabilidad espacial con zonas con mayor o menor cantidad de plantas por $\mathrm{m}^{2}$ distribuidas por toda el área de estudio, no habiendo zonas de concentración de valores para esta especie de mala hierba. El mapa de distribución espacial de Achillea millefolium (plantas por $\mathrm{m}^{2}$ ) describe un comportamiento distinto a los demás mapas de variabilidad espacial, dado que su patrón de distribución no sigue ningún patrón que sea reconocible para ningún otro mapa de variabilidad espacial.

Los mapas de variabilidad espacial de las especies de malas hierbas (Dactylis glomerata, Trifolium sp., Bellis perennis, Plantago lanceolata, Taraxacum officinale, Achillea millefolium, otras especies, densidad de individuos y número de especies) presentan zonas con mayor o menor concentración de plantas por $\mathrm{m}^{2}$. Shiratsuchi et al. (2004) describen que el comportamiento de las especies de malas hierbas en campo no ocurren de manera uniforme.

Lass \& Callihan (1993) y Colliver et al. (1996) proponen que el muestreo de las malas hierbas en el campo sea realizado haciendo el mapeado del contorno de las zonas de mayor ocurrencia de las especies de malas hierbas, generando mapas de poligonos de infestación.

Por otra parte, Lutman \& Perry (1999) describen que el muestreo localizado, como el utilizado en este estudio es el más preciso, presentando como desventaja el elevado trabajo de campo para la identificación y cuantificación de las especies de malas hierbas. Este tipo de muestreo permite la identificación individual de las especies de malas hierbas, siendo posible inferir en el proceso de gestión de las área de producción con mayor precisión, cuando se compara con la metodología de Lass \& Callihan (1993) y Colliver et al. (1996), dado que en este caso las especies no son identificadas individualmente. El mapa de variabilidad espacial del número de especies por $\mathrm{m}^{2}$ en el área de estudio, se asemeja mucho al mapa de variabilidad espacial de la $\mathrm{CE}_{\mathrm{a}}-\mathrm{V}$ y $\mathrm{CE}_{\mathrm{a}}-\mathrm{H}$, indicando que el mayor número de especies ocurre en las zonas con menores valores $\mathrm{CE}_{\mathrm{a}}$.

Se comprueba que el mapa (Figura 3) de la densidad de individuos (plantas por $\mathrm{m}^{2}$ ) y el mapa del número de especies (especies por $\mathrm{m}^{2}$ ) presentan comportamientos inversos, hecho que es confirmado por el valor de correlación lineal entre estas dos variables $(r=-0,780)$. Por otra parte, se puede decir que el mapa de 
variabilidad espacial de la densidad de individuos es muy similar al mapa de variabilidad espacial del Dactylis glomerata, confirmando el predominio de esta especie de mala hierba en el área de estudio, presentando un valor de correlación lineal de 0,890, corroborando que los mapas de variabilidad espacial de densidad de individuos y del número de especies presenten comportamiento inverso.

Debido a los bajos valores de correlación lineal (Tabla 2) entre la $\mathrm{CE}_{\mathrm{a}}-\mathrm{V}$ y $\mathrm{CE}_{\mathrm{a}}-\mathrm{H}$ y parámetros de malas hierbas (Dactylis glomerata, Trifolium sp., Bellis perennis, Plantago lanceolata, Taraxacum officinale, Achillea millefolium, otras especies, densidad de individuos y número de especies), se comprueba que no es viable el uso de la geoestatística multivariante para la mejora de la estimación de las especies de malas hierbas en el área de estudio utilizando los datos de la $\mathrm{CE}_{\mathrm{a}}-\mathrm{V}$ y $\mathrm{CE}_{\mathrm{a}}-\mathrm{H}$ como información secundaria.

La ocurrencia de las malas hierbas en la pradera demostró que la presencia de especies fue influida por el nivel freático en el área de estudio.

Se encontraron bajos valores de correlación de las medidas de $\mathrm{CE}_{\mathrm{a}}$ con las mediciones de malas hierbas en el área de estudio, estando las mismas influidas por el relieve del área.

Los mapas de variabilidad espacial para las malas hierbas demuestran la dominancia de especies (Dactylis glomerata y el Trifolium sp.) y el endemismo de otras especies (Bellis perennis, Plantago lanceolata, Taraxacum officinale y Achillea millefolium) dentro del contexto de la ecologia, tal conocimiento es importante, ya que nos permite determinar funciones de manejo que beneficien a todas las especies, lo cual es importante para las especies con riesgo de extinción.

La utilización del cokrigeado usando como variable secundaria los datos de $\mathrm{CE}_{\mathrm{a}}$ no es viable para la mejora de los mapas de malas hierbas debido a que existen bajos valores de correlación lineal.

\section{AGRADECIMIENTOS}

Al Ministerio de Assuntos Exteriores y de Cooperación (MAEC-AECID) por la Beca de
Doctorado conedida al primer autor (2006-2009). Al Ministerio de Educación y Ciencia (Proyecto CGL2005-08219-C02-02), al Ministerio de Economia y Competitividad de España (Proyecto CGL2013-47814-C2), a la Xunta de Galicia (Proyecto PGIDIT06PXIC 291062PN) y al Fondo Europeo de Desarrollo Regional (FEDER) por el financiamiento parcial de este trabajo y a la granja provincial de Gaioso-Castro de la Deputación de Lugo por permitirnos usar sus instalaciones para llevar a cabo este trabajo. Los autores agradecen también a FAPEMA (Brasil) por el apoyo financiero (Proyectos APCINTER 02587/14 y BEPP 01301/15).

\section{LITERATURA CITADA}

CAMBARDELLA, C. A. et al. Field scale variability of soil properties in central Iowa soil. Soil Sci. Am. J., v. 58, n. 5, p. 1501-1511, 1994.

COLBACH, N.; FORCELLA, F.; JOHNSON, G. Temporal trends in spatial variability of weed populations in 3 continuous no-till soybean. Weed Sci., v. 48, n. 3, p. $366-377,2000$.

COLLIVER, C. T. et al. Georeferencing wild oat infestations in small grains: Accuracy and efficiency of three weed survey techniques. In: THIRD ANNUAL CONFERENCE ON PRECISION AGRICULTURE, 1996, Madison.

Proceedings... Madison: American Society of Agronomy, 1996. p. 453-463.

FAO-ISRIC. World reference base for soil resources. Roma/Wageningen: 1994. $161 \mathrm{p}$.

GEONICS. EMD38-DD Ground Conductivity Meter-Dual Dipole Version. Ontario: 2005. 34 p.

GOLDEN SOFTWARE. Surfer: User's Guide (Versión 7.0). Golden, CO: EEUU, 1999. 679 p.

ISAAKS, E. H.; SRIVASTAVA, R. M. An introduction to applied geoestatistics. New York: Oxford University Press, 1989. $561 \mathrm{p}$.

JURADO-EXPÓSITO, M. et al. Spatial and temporal analysis of Convolvulus arvensis L. populations over four growing seasons. Eur. J. Agron., v. 21, n. 3, p. 287-296, 2004.

JURADO-EXPÓSITO, M. et al. A digital elevation model to aid geostatistical mapping of weeds in sunflower crops. Agron. Sustain. Develop., v. 29, n. 2, p. 391-400, 2009.

LASS, L. W.; CALLIHAN, R. H. GPS and GIS for weed surveys and management. Weed Technol., v. 7, n. 1, p. 249-254, 1993. 
LESCH, S. M. Sensor-directed response surface sampling designs for characterizing spatial variation in soil properties. Comp. Electr. Agric., v. 46, n. 1-3, p. 153-179, 2005.

LESCH, S. M.; RHOADES, J. D.; CORWIN. D. L. The ESAP Version 2.01r user manual and tutorial guide. Riverside: Salinity Laboratory, 2000. 153 p. (Research Report, 146)

LUTMAN, P. J. W.; PERRY, N. H. Methods of weed patch detection in cereal crops. In: THE 1999 BRIGHTON CONFERENCE - WEEDS, 1999, Brighton. Proceedings... Brighton: BCPC, 1999. p. 627-634.

McNEILL, J. D. Electrical conductivity of soils and rocks. Ontario: Geonics, 1980. 22 p. (Technical Note, TN-5)

MOSQUERA LOSADA, M. A.; GONZÁLEZ RODRIGUEZ, A.; RIGUEIRO RODRÍGUEZ, A. Ecología y manejo de praderas. Vigo: Instituto Nacional de Investigación y Tecnología Agraria y Alimentaria, 1999. 214 p.

RHOADES, J. D.; RAATS, P. C. A.; PRATHER, R. J. Effects of liquid-phase electrical conductivity, water content, and surface conductivity on bulk soil electrical conductivity. Soil Sci. Soc. Am. J., v. 40, n. 5, p. 652-655, 1976.

SAN MIGUEL AYANZ, A. Gramíneas de interés para la implantación de praderas y la revegetación de zonas degradadas. Ecología y pautas básicas de utilización. Madrid: Universidad Politécnica de Madrid, 2008. 25 p.
SANTOS, C. M. A. Estatística descritiva - Manual de Auto-aprendizagem. Lisboa: Edições Sílabo, 2007. $261 \mathrm{p}$.

SCHAFFRATH, V. R. et al. Variabilidade espacial de plantas daninhas em dois sistemas de manejo de solo. R. Bras. Eng. Agríc. Amb., v. 11, n. 1, p. 53-60, 2007.

SIQUEIRA, G. M., VIEIRA, S. R.; CAMARGO, M. B. P. Variabilidade espacial do armazenamento e da perda média diária de água pelo solo no sistema de semeadura direta em Campinas, SP. Bragantia, v. 67, n. 1, p. 213-223, 2008.

SIRATSUCHI, L. S.; MOLIN, J. P.; CRISTOFFOLETI, P. J. Mapeamento da distribuição especial da infestação de Panicum maximum durante a colheita da cultura do milho. Planta Daninha, v. 22, n. 2, p. 269-274, 2004.

VIEIRA, S. R. et al. Handbook for geoestatistical analysis of variability in soil and climate data. Tópicos Ci. Solo, v. 2, n. 1, p. 1-45, 2002.

VILLARÍAS, J. L. Atlas de Malas Hierbas. Madrid: Grupo Mundi-Prensa, 2000. 536 p.

WARRICK, A. W.; NIELSEN, D. R. Spatial variability of soil physical properties in the field. In: HILLEL, D. Applications of soil physics. New York: Academic Press, 1980. 